\title{
PHÂN LÂP HỢP CHẤT FUB-AMB TRONG GIÁM ĐİNH PHÁP Y CÁC MẪU CẦN SA TỔNG HỢP THU TẠI VIÊTT NAM TỪ 2016 - 2018
}

\section{TÓM TẮT}

Đặt vấn đề: Nhóm cần sa tổng hợp, còn được gọi là "cỏ Mỹ" là nhóm ma túy có chứa nhiều chất rất nguy hiểm, làm phát sinh các hành vi vi phạm pháp luật, điển hình như chất FUB-AMB. Trong bài báo này hợp chất FUB-AMB lần đầu tiên tại Việt Nam, được phân lâp và xác định cấu trúc bằng các phương pháp phân tích phổ học (UV, IR, ${ }^{1} \mathrm{H}-\mathrm{NMR},{ }^{13} \mathrm{C}-\mathrm{NMR}$ ). Mục tiêu: Phân lập và tinh khiết hóa hợp chất FUB-AMB dùng làm chất chuẩn định tính-định lượng. Đối tượng và phương pháp: Mẫu cần sa tổng hợp dạng cỏ Mỹ được thu thập trong các vụ án tại Việt Nam từ năm 2016 đến 2018, sau đó được đun cách thủy với methanol ở nhiệt độ $40^{\circ} \mathrm{C}$ thu được cao methnol. Phân lập các phân đoạn cao toàn phần thu được FUB-AMB bằng sắc ký cột hay sắc ký điều chế. Kết quả: Từ $10 \mathrm{~g}$ cao methanol toàn phần thu được hợp chất FUB-AMB với độ tinh khiết 98,34\% trên HPLC. Kết luận: Từ cao methanol toàn phân bằng kỹ thuật sắc ký đã phân lập được hợp chất FUB-AMB 98,34\%. Từ kết quả này chẩt FÜB-AMB có thể làm chất chuẩn trong kiểm nghiệm, góp phân xây dựng phương pháp phân tích các chất ma túy trong nhóm cần sa tông hợp, hỗ trợ công tác điều tra, truy tố, xét xử tội phạm ma túy của cơ quan chức năng.

Tư khóa: Cần sa tổng hợp, FUB-AMB (FA), sắc ký cột, HPLC

\section{SUMMARY \\ ISOLATION OF FUB-AMB FROM SYNTHETIC CANNABINOIDS IN HERBAL MIXTURES AT VIETNAM IN THE PERIOD OF 2016 - 2018}

Backgrounds: Synthetic cannabinoids are currently the largest group of new psychoactive substances and one of the most recent synthetic cannabinoids that have appeared in Vietnam is FUBAMB. In this study, the first FUB-AMB compound in Vietnam was isolated and structure determined by spectroscopic analytical methods (UV, IR, ${ }^{1} \mathrm{H}-\mathrm{NMR}$, $\left.{ }^{13} \mathrm{C}-\mathrm{NMR}\right)$. Subjects and methods:: Isolation and purity of FUB-AMB compounds used as working standard (substance). The samples of synthetic cannabinoids were collected from cases in Vietnam from 2016 to 2018, and then watered with methanol at $40^{\circ} \mathrm{C}$, recovered solvent to get total methanol extract. Isolation of fractions from methanol extract by column chromatography to collect FUB-AMB. Results:

*Cao đẳng Bách khoa Nam Sài Gòn

**Phân viên Khoa hoc hình sư TPHCM

Chịu trách nhiệm chính: Nguyễn Anh Tuấn

Email: huynhtuan9000@gmail.com

Ngày nhận bài: 2/12/ 2020

Ngày phản biên khoa hoc: 14/1/2021

Ngày duyệt bài: 20/2/2021

\section{Nguyễn Anh Tuấn*, Nguyễn Đăng Tiến**}

FUB-AMB was collected from $10 \mathrm{~g}$ of total metanol extract with the purity were $98,34 \%$. Conclusions: By chromatography methods, the compound FUB-AMB (1g) was isolated. From this result, FUB-AMB can be used as a standard substance in testing, contributing to the development of a method for analyzing narcotic drugs in the synthetic cannabinoids to support the drug crimes handling of the authorities.

Key words: Synthetic cannabinoids, FUB-AMB, column chromatography, HPLC

\section{I. ĐĂTT VẤN ĐỀ}

Từ năm 2016 đến 2018, Viện Khoa học Hình sự đã phát hiện ra nhiều hợp chất cần sa tổng hợp mới ở Việt Nam và một trong những chất gần đây nhất phát hiện trong cỏ Mỹ là FUB-AMB, chất này gây ảo giác và có tác dụng độc hại như gây ức chế hô hấp, ngừng tim, thiếu máu não, co giật. Sau khi danh sách cấm các hợp chất cần sa tổng hợp mới, bao gồm FUB-AMB được Chính phủ ban hành trong danh mục các chất ma túy cần kiểm soát theo Nghị định số 73/2018/NĐ-CP, và Nghị định số 60/2020/NĐ-CP của chính phủ ${ }^{[1]}$, một trong những khó khăn lớn nhất trong công tác thụ lý các vụ án ma túy là thiếu chất chuẩn phục vụ cho công tác kiểm nghiệm.

Trên thế giới, hợp chất FUB-AMB được xác định qua các nghiên cứu của Cơ quan phòng chống tôii pham liên minh Châu Âu; tổ chức y tế thế giới; Samuel D. Banister và cống sự và Ivo D. Ivanov bằng phương pháp sắc ký khí ghép với đầu dò phổ khối (GC-MS) là phương pháp thường được sử dụng để phân tích các mẫu FUB-AMB [2] [3] [7]. Đầu dò khối phổ là đầu dò vạn năng, có thể đồng thời định danh nhanh và định lượng chính xác được hàm lượng FUB-AMB, hê thống máy GC-MS được trang bị tại các phòng thí nghiệm giám định ma túy tại Việt Nam nên tạo thuận lợi cho công tác phân tích. Tuy nhiên, chuẩn FUB-AMB ở Việt Nam thì không có sẵn, phải mua từ nước ngoài với giá thành rất cao và về tính pháp lý rất khó mua hợp chất này, vì thế, gây không ít khó khăn cho công tác kiểm nghiệm. Xuất phát từ các lý do trên, nghiên cứu này được thực hiện tại Phân Viện Khoa học Hình sự TP. HCM nhằm phân lập và xác định cấu trúc cũ̃ng như tinh khiết hóa FUB-AMB từ cỏ Mỹ thu được trong các vụ án về ma túy ở Viêt Nam nhẳm sử dụng chất phân lập này như chất chuẩn định tính, định lượng trong các phòng thí nghiệm giám định trên toàn quốc. 
II. ĐỐI TƯỢNG VÀ PHƯƠNG PHÁP NGHIÊN CỨU

2.1. Nguyên liệu. Nguồn gốc: Mẫu "cỏ Mỹ" thu thập từ các vụ án tại Việt Nam từ năm 2016 đến 2018.

Mô tả: Mẫu "cỏ Mỹ" được đóng gói dưới dạng các gói thực vật khô, cắt nhỏ, tẩm mùi thơm đặc trưng, nhưng thực chất "cỏ Mỹ" là hỗn hợp cây cỏ được tẩm ướp hoạt chất ma túy cần sa tổng hợp.

\subsection{Hóa chất, dung môi}

Bản mỏng tráng sẵn pha thường silica gel F254 (Merck), chất hấp phụ silica gel pha thường (cõ hạt 40-63 $\mu \mathrm{m}$, Merck). Dung môi n-hexan, ethyl acetat (EtOAc), cloroform $\left(\mathrm{CHCl}_{3}\right)$, methanol $(\mathrm{MeOH})$, nước cất $\left(\mathrm{H}_{2} \mathrm{O}\right)$.

\subsection{Thiết bị, dụng cụ}

- Đèn UV 2 bước sóng $254 \mathrm{~nm}$ và $365 \mathrm{~nm}$ (Vilber Lourmat CN-15-CL).

- Máy đo phổ bức xạ UV - Vis Cintra 10e tại Phân viên khoa học hình sự TP. HCM.

- Máy đo phổ hồng ngoại (IR) FT-IR Spectrophotometer (Perkin Elmer, Mỹ) tại Viện Khoa học Vật liệu Ứng dụng - Viện Hàn lâm Khoa học và Công nghệ Việt Nam

- Máy đo phổ khối Agilent Máy GC/MS Agilent 7890B tại Phân viện khoa học hình sự TP. HCM Máy đo phổ cộng hưởng từ hạt nhân (1H-NMR, 13C-NMR) Bruker AM500 FT-NMR tại Đại học Khoa hoc tự nhiên TP. HCM.

- Máy sắc ký lỏng hiệu năng cao (HPLC) Shimadzu LC - 20AD/PDA tại Viện kiểm nghiệm thuốc TP. HCM.

- Máy phân tích nhiêt lượng vi sai DSC Q200 V24.9 Build 121 Viện Khoa học Vật liệu Ứng dụng - Viện Hàn lâm Khoa học và Công nghệ VN

- Cột sắc ký và các dụng cụ thủy tinh phòng thí nghiệm.

\subsection{Phương pháp nghiên cứu}

Chiết xuất và phân lập. Lựa chọn dung môi chiết xuất FUB - AMB: Methanol, ethyl acetat, acetonitril, dicloromethan là các dung môi hòa tan tốt FUB - $A M B$ để chiết được hoàn toàn chất phân tích từ nền mẫu cỏ Mỹ, loại bỏ tối đa ảnh hưởng của nền mẫu, chất phân tích bền trong môi trường pha mẫu.

Mẫu cỏ Mỹ được được sấy khô ở nhiệt độ $50^{\circ} \mathrm{C}$, rồi được nghiền nhỏ thành bột mịn $(2,1 \mathrm{~kg})$ rồi cách thủy với methanol (3 lần, mỗi lần 5 lít). Dịch chiết được lọc qua giấy lọc và gộp lại, cô dịch chiết bằng cất quay dưới áp suất giảm thu được $300 \mathrm{~g}$ cao methanol.

Cao toàn phần $(10 \mathrm{~g})$ được hòa với lượng tối thiểu với methanol, trộn với silcagel và tiến hành sắc ký cột với chất hấp phụ silicagel pha thường, giải hấp bằng dung môi $100 \%$ n-hexan; $100 \%$ $\mathrm{CHCl}_{3}$ và hệ dung môi $\mathrm{n}$-hexan: ethyl acetat (tỷ lệ 9:1 và 8:2) thu được 4 phân đoạn ký hiệu là 1 $\rightarrow$ 4. Các phân đoạn sau khi tinh chế thu được hợp chất đă̆t tên là $F A(1 \mathrm{~g})$.

Xác định cấu trúc các chất phân lập

- Kiểm tra độ tinh khiết của hợp chất phân lập được bằng SKLM và HPLC.

- Xác định cấu trúc của hợp chất FA tinh khiết phân lập được bằng các phương pháp phổ nghiệm như UV, IR, MS, NMR.

\section{KẾT QUẢ NGHIÊN CỨU}

3.1. Kết quả chiết xuất và phân lập. Cân khoảng $0,01 \mathrm{~g}$ cần sa tổng hợp rồi hòa với $5 \mathrm{ml}$ dung môi, vortex 5 phút, sau đó siêu âm 10 phút, lọc qua màng lọc $0,45 \mu \mathrm{m}$. Thực hiện cùng điêu kiện với 4 loại dung môi: methanol, ethyl acetat, acetonitril, dicloromethan, kết quả diện tích pic trên GC/MS tại thời gian lưu 9,6 phút được trình bày như bảng 1 . Từ đó, lựa chọ dung môi methanol cho quá trình chiết mẫu do cho kết quả diện tích pic lớn nhất.

Bảng 1. Kềt quả xử lý với bốn loại dung môi

\begin{tabular}{|c|c|c|}
\hline Dung môi & $\begin{array}{c}\text { Khối lượng } \\
\text { cân } \mathbf{( g )}\end{array}$ & $\begin{array}{c}\text { Diệ̣n tích pic } \\
\text { ( } \mathbf{\mu V . m i n )}\end{array}$ \\
\hline Methanol & 0,0104 & $21.352 .718,53$ \\
\hline Ethyl acetat & 0,0105 & $20.519 .707,73$ \\
\hline Acetonitril & 0,0104 & $15.093 .683,49$ \\
\hline Dicloromethan & 0,0106 & $7.418 .441,01$ \\
\hline
\end{tabular}

Điều kiện sắc ký: Cột HP - 5ms Ultra Inert $60^{\circ} \mathrm{C}-325^{\circ} \mathrm{C}(30 \mathrm{~m} \times 250 \mu \mathrm{m} \times 0,25 \mu \mathrm{m})$. Khí mang: khí Heli, tốc độ dòng: 1,2 mL/phút. Detector MS, chế độ Scan nhiệt độ detector: $280^{\circ} \mathrm{C}$. Nhiệt độ tiêm mẫu: $280^{\circ} \mathrm{C}$, tỷ lệ chia dòng $1: 10$, thể tích tiêm: $1 \mu \mathrm{L}$. Chương trình nhiệt: Nhiệt độ ban đầu $150^{\circ} \mathrm{C}$ lưu giữ trong 1 phút, tăng lên $290^{\circ} \mathrm{C}$, tốc độ tăng nhiệt là $30^{\circ} \mathrm{C} /$ phút, lưu giữ trong 4 phút.
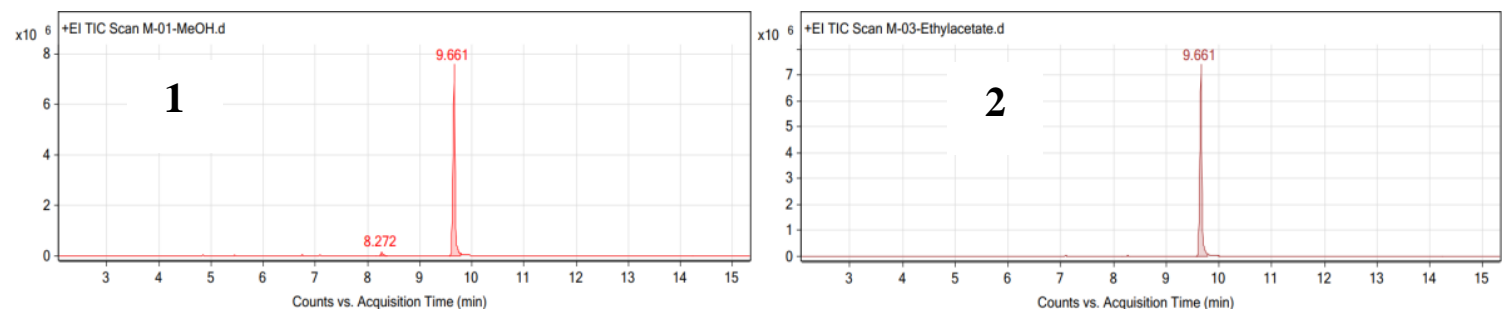

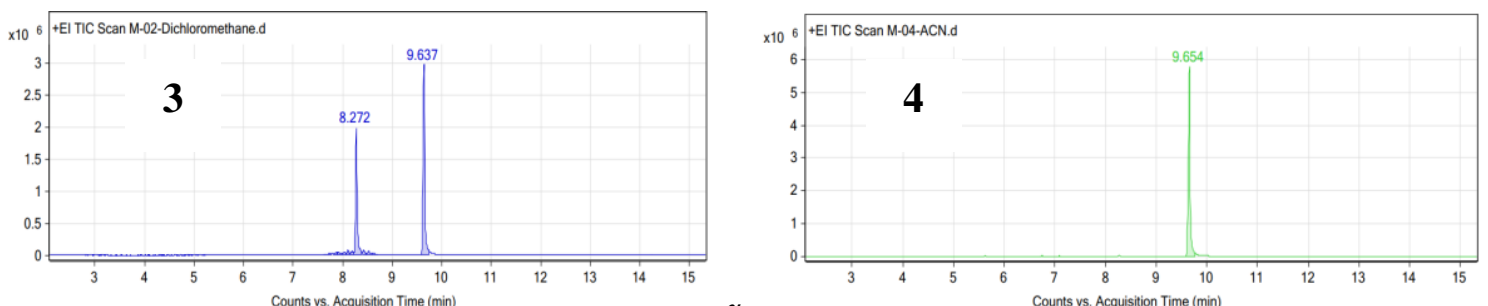

Hinh 1. Sắc ký đồ của mẫu cỏ Mỹ với 4 loại dung môi

(1. Methanol; 2. Ethylacetat; 3. Dicloromethan; 4. Acetonitril)

Sau khi chiết với methanol và tiến hành sắc ký cao methanol trên cột sắc ký thu được 4 phân đoạn. Các phân đoạn từ 1 đến 4 được kiểm tra tinh khiết trên SKLM.

Tại phân đoạn n-hexan - ethylacetat (8:2) (phân đoạn số 4) sau khi cô quay thu hồi dung môi thu được tinh thể hình kim đặt tên là FA. Hợp chất $\mathrm{FA}$ được tinh chế bằng cách rửa tinh thể với dung môi methanol lạnh sau đó hòa với dung môi thích hợp và để kết tinh ở nhiệt độ phòng.

Với khối lượng $1 \mathrm{~g}$ hợp chất FA được kiểm tra độ tinh khiết bằng HPLC với đầu dò PDA tại Viện kiểm nghiệm thuốc thành phố Hồ Chí Minh cho kết quả độ tinh khiết là 98,34\%.

3.2. Kết quả xác định câu trúc hợp chất phân lập

- Sắc ký lớp mỏng: với hệ dung môi n-hexan - ethylacetat (8:2); trên bản mỏng silica gel F254 (Merck), hợp chất FA cho một vết có hình tròn, màu sắc hơi tím. Phát hiện vết ở UV $254 \mathrm{~nm}$, UV $365 \mathrm{~nm}$ trên ba hệ dung môi khác nhau như hình 4.

- Phổ UV/MeOH có 2 đỉnh hấp thu cực đại tại bước sóng $209 \mathrm{~nm}$ và $299 \mathrm{~nm}^{[5]}$

- Phổ IR/KBr, số sóng $\left(\mathrm{cm}^{-1}\right): 3422(\mathrm{w}) \mathrm{N}-\mathrm{H}$ amin; 1740 (s) $\mathrm{C}=\mathrm{O}$ amid; 1674 (s) $\mathrm{C}=\mathrm{N}$ vòng indazol; 1534 (s) C=O ester; 1218 (s) C-N; 1181 (s) C-F; $781(\mathrm{~m}) \mathrm{C}-\mathrm{H}$ vòng thơm ${ }^{[5][6]}$

- Phổ MS (Hình 5): phổ FA cho các mảnh có $\mathrm{m} / \mathrm{z}=109,0 ; \mathrm{m} / \mathrm{z}=253,1 ; \mathrm{m} / \mathrm{z}=269,1 ; \mathrm{m} / \mathrm{z}=$ 324,2 và $\mathrm{m} / \mathrm{z} 383,2$ hoàn toàn phù hợp với công thức phân tử $\mathrm{C}_{21} \mathrm{H}_{22} \mathrm{FN}_{3} \mathrm{O}_{3}$, có số khối chính xác là $383,2 .{ }^{[5]}$

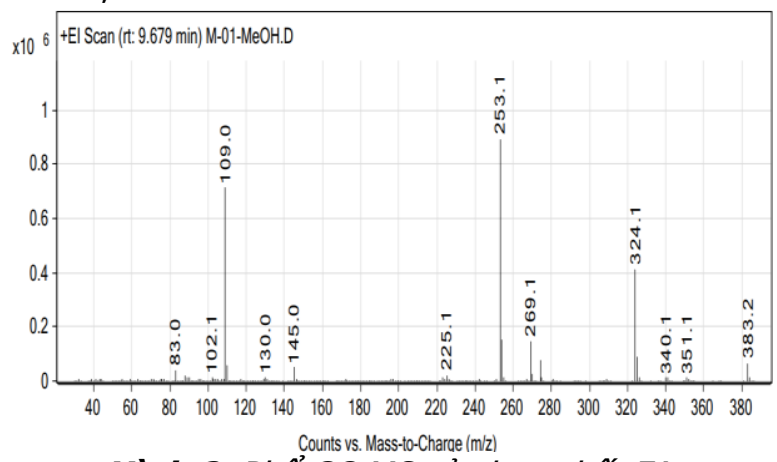

Hình 2. Phố GC-MS cúa hợp chất FA

- Phổ NMR: Hợp chất FA được đo phổ NMR (DMSO, $500 \mathrm{MHz}$ ) và so sánh với dữ liệu phổ tương ứng của FUB-AMB $\left(\mathrm{CDCl}_{3}, 500 \mathrm{MHz}\right)$ trong tài liệu tham khảo. Kết quả cho thấy các dữ liệu phổ NMR của FA hoàn toàn phù hợp với FUBAMB (Bảng 2).

Bảng 2. So sánh dữ liệu phổ 13C-NMR, 1H-NMR của FA và FUB-AMB

\begin{tabular}{|c|c|c|c|c|}
\hline \multirow[b]{2}{*}{ Vị trí } & \multicolumn{2}{|r|}{ FA } & \multicolumn{2}{|c|}{ FUB - AMB (Samuel D. Banister) ${ }^{[4]}$} \\
\hline & $\begin{array}{c}{ }^{13} \mathrm{C}-\mathrm{NMR} \\
(125 \mathrm{MHz})\end{array}$ & $\begin{array}{c}\text { 1'H-NMR } \\
(500 M H z)\end{array}$ & $\begin{array}{l}{ }^{13} \mathrm{C}-\mathrm{NMR} \\
(75 \mathrm{MHz})\end{array}$ & $\begin{array}{c}\text { 1H-NMR } \\
(300 M H z) \\
\end{array}$ \\
\hline $1^{\prime}$ & 162.6 & - & 162.6 & - \\
\hline 3 & 136.9 & - & 137.5 & - \\
\hline $3 a$ & 122.4 & 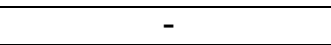 & 123.1 & - \\
\hline 4 & 121.7 & $8.15,1 \mathrm{H}, \mathrm{J}=8 \mathrm{~Hz}$ & 123.0 & $8.36,1 \mathrm{H}, \mathrm{J}=7.8 \mathrm{~Hz}$ \\
\hline 5 & 122.7 & $7.29,1 \mathrm{H}, \mathrm{t}, \mathrm{J}=7 \mathrm{~Hz}$ & 123.5 & $7.0,2 \mathrm{H}, \mathrm{t}, \mathrm{J}=7.8 \mathrm{~Hz}$ \\
\hline 6 & 127.0 & $7.45,1 \mathrm{H}, \mathrm{m}$ & 127.2 & \\
\hline 7 & 110.5 & $7.78,1 \mathrm{H}, \mathrm{J}=8.5 \mathrm{~Hz}$ & 109.6 & $7.5,1 \mathrm{H}, \mathrm{J}=9.0 \mathrm{~Hz}$ \\
\hline $7 a$ & 140.5 & - & 140.9 & - \\
\hline $1^{\prime \prime}$ & 51.8 & $5.78,2 \mathrm{H}, \mathrm{s}$ & 53.1 & $5.61,2 \mathrm{H}, \mathrm{s}$ \\
\hline $2^{\prime \prime}$ & 132.9 & - & 131.9 & \\
\hline $3^{\prime \prime} / 7^{\prime \prime}$ & 129.4 & $7.32-7.35,2 \mathrm{H}, \mathrm{m}$ & 129.1 & $\begin{array}{c}7.27-7.42,2 \mathrm{H}, \mathrm{m} \\
7.31,1 \mathrm{H}, \mathrm{d}, \mathrm{J}=8.5,2.1 \mathrm{~Hz}\end{array}$ \\
\hline $4^{\prime \prime} / 6^{\prime \prime}$ & 115.5 & $7.14-7.18,2 \mathrm{H}, \mathrm{m}$ & 116.0 & $\begin{array}{c}7.19-7.23,2 \mathrm{H}, \mathrm{m} \\
7.15,1 \mathrm{H}, \mathrm{J}=7.9 \mathrm{~Hz}\end{array}$ \\
\hline
\end{tabular}




\begin{tabular}{|c|c|c|c|c|}
\hline $5^{\prime \prime}$ & 161.2 & - & 162.5 & - \\
\hline $1^{\prime \prime \prime}$ & 171.9 & - & 172.7 & - \\
\hline $2^{\prime \prime \prime}$ & 57.3 & $4.45,1 \mathrm{H}, \mathrm{dd}, \mathrm{J}=7,1 \mathrm{~Hz}$ & 56.9 & $4.83,1 \mathrm{H}, \mathrm{dd}, \mathrm{J}=8.7,5.1 \mathrm{~Hz}$ \\
\hline $3^{\prime \prime \prime}$ & 29.9 & $2.26,1 \mathrm{H}, \mathrm{m}$ & 31.8 & $2.3,1 \mathrm{H}, \mathrm{m} \mathrm{J}=6.6 \mathrm{~Hz}$ \\
\hline $4^{\prime \prime \prime}$ & 19.0 & $0.97,3 \mathrm{H}, \mathrm{d}, \mathrm{J}=6.5 \mathrm{~Hz}$ & 19.3 & $1.05,6 \mathrm{H}, \mathrm{t}, \mathrm{J}=5.4 \mathrm{~Hz}$ \\
\hline $5^{\prime \prime \prime}$ & 18.6 & $0.95,3 \mathrm{H}, \mathrm{d}, \mathrm{J}=6.5 \mathrm{~Hz}$ & 18.2 & \\
\hline $\mathrm{OCH}_{3}$ & 51.6 & $0.945-0.977,3 \mathrm{H}, \mathrm{m}$ & 52.3 & $3.78,3 \mathrm{H}, \mathrm{s}$ \\
\hline $\mathrm{CONH}$ & - & $7.78,1 \mathrm{H}, \mathrm{J}=8.5 \mathrm{~Hz}$ & & $7.5,1 \mathrm{H}, \mathrm{J}=9.0 \mathrm{~Hz}$ \\
\hline
\end{tabular}

Chú thích s: singlet, d: doublet, m: multiplet

- Phổ DSC: Kết quả xác định nhiệt độ nóng chảy bằng phương pháp DSC cho thấy FA có điểm chảy tại $121,8^{\circ} \mathrm{C}$.

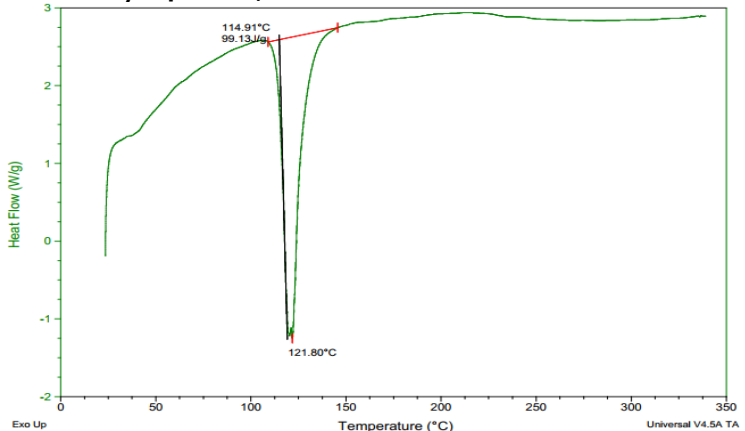

Hình 3. Phổ DSC của hợp chất FA

Nhận xét: Từ kết quả trên, các dữ liệu phổ đều cho thây cấu trúc sản phẩm phân lập hoàn toàn phù hợp với cấu trúc của FUB-AMB có công thức cấu taoo như hình 7.<smiles>COC(=O)[C@H](NC(=O)c1nn(Cc2ccc(F)cc2)c2ccccc12)C(C)(F)F</smiles>

trên 98\%. Cấu trúc của hợp chất phân lập cũng đã được khẳng định bằng phương pháp phổ học như UV - Vis, IR, GC-MS, DSC trong đó phổ NMR đã được biện giải và so sánh với các tài liệu tham khảo cho kết quả hợp chất phân lập được là FUB-AMB. Sản phẩm FUB-AMB có độ tinh khiết trên $98 \%$ nên được sử dụng như chất chuẩn làm việc để phục vụ cho các bước nghiên cứu tiếp theo trong việc xây dựng và thẩm định quy trình định lượng các mẫu cỏ Mỹ tại Việt Nam.

\section{KẾT LUÂ̂N}

Trong những năm 2016 - 2018, các chất ma túy trong cân sa tổng hợp thường gặp trong các vụ án là hợp chất FUB-AMB. Phân tích các dữ kiện phổ và so sánh với những tài liệu đã công bổ, hợp chất FA được phân lập là FUB-AMB. Đề tài đã phân lập được một chất bột màu trắng tan tốt trong $\mathrm{MeOH}$, ethyl acetat, acetonitil, không tan trong nước có công thúc phân tử $\mathrm{C}_{21} \mathrm{H}_{22} \mathrm{FN}_{3} \mathrm{O}_{3}$ có độ tinh khiết $98,34 \%$ góp phần phong phú thêm nguồn chất chuẩn để kiểm nghiệm các hợp chất trong các mẫu vật chứa cần sa tổng hợp.

\section{TÀI LIÊU THAM KHẢO}

1. Nghị định Sửa đổi, bổ sung Danh mục các chất ma túy và tiên chất ban hành kèm theo Nghị định số 73/2018/NĐ-CP ngày 15 tháng 5 năm 2018 của Chính phủ quy định các danh mục chất ma túy và tiền chất, 60/2020/NĐ-CP, ngày 29 tháng 5 năm 2020.

2. Ivo D. Ivanov (2019), "A case of $5 f-A D B$ / FUBAMB abuse: druginduced or drug-related death", Forensic Science International, pp $372-377$.

Hinh 4. Công thức cấu tạo của $F A$

\section{BÀN LUÂN}

Khi nghiên cứu cấu trúc và độ tan của FUB$\mathrm{AMB}$, nhận thấy hợp chất này tan tốt nhất trong $\mathrm{MeOH}$ nền lựa chọn dung môi $\mathrm{MeOH}$ để chiết cao toàn phần từ mẫu cỏ Mỹ. Bước tiếp theo, đề tài đã khảo sát được các hệ dung môi có độ phân cực khác nhau để tách FUB-AMB trên sắc ký cột, khảo sát pha động để kiểm tra các phân đoạn trên sắc ký lớp mỏng. Tinh thể từ phân đoạn dự đoán có chứa FUB-AMB đã được xác định độ tinh khiết trên HPLC/PDA cho kết quả

3. Prevention of and Fight against Crime programme (2015), "Analytical report: FUB-AMB $\left(\mathrm{C}_{21} \mathrm{H}_{22} \mathrm{FN}_{3} \mathrm{O}_{3}\right)^{\prime \prime}$, European Commission.

4. Samuel D. Banister (2016), "Pharmacology of Valinate and tert-Leucinate Synthetic Cannabinoids 5F-AMBICA, 5F-AMB，5F-ADB， AMB-FUBINACA, MDMB-FUBINACA, MDMB-CHMICA, and Their Analogues", ACS Chemical Neuroscience, pp 1241-1254

5. The New Zealand Ministry of Health (2018), AB-FUBINACA and AMB FUBINACA: Report to the Expert Advisory Committee on Drugs, pp 1-13.

6. World Health Organization (2018), Critical Review Report: FUB-AMB, pp 5-19. 